\title{
An application of nonstationary wavelets
}

Junjian Zhao*

\section{"Correspondence:}

zhaojunjian@emails.bjut.edu.cn

Department of Mathematics, Tianjin

Polytechnic University, No. 399

Binshuixi Street, Xiqing District,

Tianjin, 300387, P.R. China

\begin{abstract}
Using nonstationary wavelets, we investigate the wavelet expansion in the standard Besov spaces. Especially, the nonstationary wavelets' characterization for Besov spaces is given.
\end{abstract}

MSC: 42C40; 35Q30; 41A15

Keywords: Besov spaces; nonstationary wavelet; characterization

\section{Introduction}

In this paper, we shall study the nonstationary wavelet [1] expansion in the standard Besov spaces. Nonstationary wavelet systems are generally obtained from a sequence of nonstationary refinable functions.

Definition 1.1 A sequence of functions $\left\{\phi_{j-1}\right\}_{j \in N}$ in $L_{2}(R)$ is said to consist of nonstationary refinable functions if, for all $j \in N$,

$$
\widehat{\phi}_{j-1}(\xi)=\widehat{a}_{j}\left(\frac{\xi}{2}\right) \widehat{\phi}_{j}\left(\frac{\xi}{2}\right), \quad \text { a.e. } \xi \in R
$$

where $\widehat{a}_{j}$ are $2 \pi$-periodic measurable functions, called refinement masks, or simply masks.

The classical Fourier transform is defined by $\widehat{f}(\xi):=\int_{R^{n}} f(x) e^{-i x \xi} d x$ for $f \in L_{1}$. The standard extension can be made to $L_{2}$ functions. Wavelet functions $\psi_{j-1}^{l}, j \in N$ and $l=$ $1,2, \ldots, Z_{j}$ (quite often $Z_{j}=3$ ), are generally obtained from nonstationary refinable functions by

$$
\widehat{\psi}_{j-1}^{l}(\xi)=\widehat{b}_{j}^{l}\left(\frac{\xi}{2}\right) \widehat{\phi}_{j}\left(\frac{\xi}{2}\right), \quad j \in N, l=1,2, \ldots, Z_{j},
$$

where $\widehat{b}_{j}^{l}(\xi)$ are $2 \pi$-periodic measurable functions called wavelet masks. The masks $\widehat{a}_{j}$ and $\widehat{b}_{j}^{l}$ satisfy $\left|\widehat{a}_{j}(\xi)\right|^{2}+\sum_{l=1}^{Z_{j}}\left|\widehat{b}_{j}^{l}(\xi)\right|^{2}=1$. When $Z_{j}=3$, let $\widehat{b}_{j}^{l}(\xi):=e^{-i \xi} \overline{\widehat{a}_{j}(\xi+\pi)}, \widehat{b}_{j}^{2}(\xi):=$ $2^{-1} A_{j}(\xi)+e^{-i \xi} \overline{A_{j}(\xi)}$ and $\widehat{b}_{j}^{3}(\xi):=2^{-1} A_{j}(\xi)-e^{-i \xi} \overline{A_{j}(\xi)}$, where $A_{j}(\xi):=1-\left|\widehat{a}_{j}(\xi)\right|^{2}-\mid \widehat{a}_{j}(\xi+$

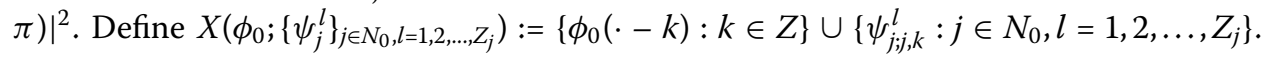
Then the following theorem holds.

Theorem 1.1 (i) (Theorem 1.3, [1]). Let $\widehat{a}_{j}(\xi):=\widehat{a}_{m_{j}, l_{j}}^{I}$ (mask for the pseudo-spline of type I with order $\left.\left(m_{j}, l_{j}\right)\right)$. Then $X\left(\phi_{0} ;\left\{\psi_{j}^{l}\right\}_{j \in N_{0}, l=1,2,3}\right)$ is a compactly supported and $C^{\infty}$ wavelet frame in $H_{2}^{s}(R)$ for arbitrary $s>0$;

\section{Springer}

(02014 Zhao; licensee Springer. This is an Open Access article distributed under the terms of the Creative Commons Attribution License (http://creativecommons.org/licenses/by/2.0), which permits unrestricted use, distribution, and reproduction in any medium, provided the original work is properly cited. 
(ii) (Theorem 1.4, [2]). When $\widehat{a}_{j}:=\widehat{a}_{m_{j}, m_{j}}^{I}$, then $X\left(\phi_{0} ;\left\{\psi_{j}\right\}_{j \in N_{0}}\right)\left(Z_{j}=1\right)$ is a compactly supported orthonormal basis in $L_{2}(R)$, and $\psi_{j}\left(Z_{j}=1\right)$ has $m_{j+1}$ vanishing moments. More precisely, Supp $\phi_{j} \subset[-L, L]$ and Supp $\psi_{j} \subset[-L, L]$ with uniform constant $L>0$.

In this paper, we use $H_{2}^{s}(R)$ to denote the classical $L_{2}$-Sobolev spaces with the smoothness parameter $s$. It is well known that Besov spaces contain a large number of fundamental spaces, such as Sobolev spaces, Hölder spaces, Lipschitz spaces etc. [3, 4]. They are frequently used in certain PDEs as the solution spaces. To extend the result of (i) in Theorem 1.1, we shall characterize Besov spaces by using nonstationary wavelets in this paper. It should be pointed out that Bittner and Urban [5] study the following standard Besov spaces: Let $0<p, q \leq \infty, s>0$, and let $[s]$ stand for the largest integer less than or equal to $s$,

$$
B_{p, q}^{s}\left(R^{n}\right):=\left\{f \in L_{p}\left(R^{n}\right):|f|_{B_{p, q}^{s}\left(R^{n}\right)}<\infty\right\} .
$$

Here, $|f|_{B_{, q}^{s}\left(R^{n}\right)}:=\left\|\left(2^{j s} \omega_{p}^{M}\left(f, 2^{-j}\right)\right)_{j \in Z}\right\|_{\ell_{q}}$ with $M \geq[s]+1$ and $\omega_{p}^{M}\left(f, 2^{-j}\right)$ denotes the $M$ th order smooth modulus of a function $f$, defined by $\sup _{|h| \leq 2^{-j}}\left\|\Delta_{h}^{M} f(\cdot)\right\|_{L_{p}\left(R^{n}\right)}$ as usual. The classical difference operator $\Delta_{h}$ is defined by $\Delta_{h} f(\cdot):=f(\cdot+h)-f(\cdot)$, as well as $\Delta_{h}^{M} f=$ $\Delta_{h}\left(\Delta_{h}^{M-1} f\right)$ for a positive integer $M>1$. The Besov (quasi-)norm is given by $\|f\|_{B_{p, q}^{s}\left(R^{n}\right)}:=$ $\|f\|_{L_{p}\left(R^{n}\right)}+|f|_{B_{p, q}^{s}\left(R^{n}\right)}$ and the two integers $M, M^{\prime}>s$ yield equivalent norms ([6], Remark 3.2.2).

Based on Hermite multiwavelets, Bittner and Urban characterize $B_{p, q}^{s}$ by using sequence norms,

$$
\|a\|_{\ell_{p}}:=\left\|\left(a_{j_{0}, k}\right)_{k \in Z^{n}}\right\|_{\ell_{p}}, \quad\|b\|_{\ell_{p, q}^{s}}:=\left\|\left(2^{j\left(s+\frac{n}{2}-\frac{n}{p}\right)}\left\|\left(b_{j, k}\right)_{k \in Z^{n}}\right\|_{\ell_{p}}\right)_{j \geq j_{0}}\right\|_{\ell_{q}}
$$

for $a=\left(a_{k}\right)_{k \in Z^{n}} \in \ell_{p}, b=\left(b_{j, k}\right)_{j \geq j_{0}, k \in Z^{n}} \in \ell_{p, q}^{s}$. However, due to the regularity restrictions of the Hermite splines, their characterization requires $\frac{1}{p}<s<\min \left\{3,1+\frac{1}{p}\right\}$ in the quadratic case and $1+\frac{1}{p}<s<\min \left\{4,2+\frac{1}{p}\right\}$ in the cubic one (e.g. [5, 7, 8]). In [9], we remove that restriction of $s$ by using the B-spline wavelets with weak duals as introduced in [10], but the supports of the wavelets become larger as $s$ increases. So, the main result of this paper is to characterize Besov spaces via nonstationary wavelets because of their arbitrary smoothness and uniform support.

Let $N, Z$, and $R$ be the set of positive integers, the set of integers, and the set of real numbers, respectively, as well as $N_{0}:=N \cup\{0\}$. Throughout this paper, we use $A \preceq B$ to abbreviate that $A$ is bounded by a constant multiple of $B, A \succeq B$ is defined as $B \preceq A$ and $A \sim B$ means $A \preceq B$ and $B \preceq A$. Write

$$
\langle f, g\rangle=\int_{\Omega} f(t) \overline{g(t)} d t
$$

for $f \in L_{p}(\Omega), g \in L_{p^{\prime}}(\Omega)$ with Lebesgue measurable set $\Omega \subseteq R^{n}, \frac{1}{p}+\frac{1}{p^{\prime}}=1$, and $1 \leq p \leq \infty$. For a Lebesgue measurable function $f$, the support of $f$ means the set $\operatorname{Supp}(f):=\{x \in$ $R: f(x) \neq 0\}$, which is well defined up to a set of measure 0 . Define $f_{j, k}(\cdot):=2^{\frac{j}{2}} f\left(2^{j} \cdot-k\right)$ throughout this paper.

Now, we state the Main Theorem of this paper. 
Main Theorem Let $1 \leq p \leq 2,0<q \leq \infty$; $\phi_{j}$ and $\psi_{j}$ are from (ii) of Theorem 1.1. When $f=\sum_{k} c_{0, k} \phi_{0 ; 0, k}+\sum_{j=0}^{\infty} \sum_{k \in Z} d_{j, k} \psi_{j ; j, k}$, then

$$
\|f\|_{B_{p, q}^{s}(R)} \preceq\|c\|_{l_{p}}+\|d\|_{l_{p, q}^{s}}
$$

for $s>0$. Moreover, when $m=\inf _{j} m_{j+1}, c_{0, k}:=\left\langle f, \phi_{0 ; 0, k}\right\rangle, d_{j, k}:=\left\langle f, \psi_{j ; j, k}\right\rangle$ with $j \in N_{0}$ and $k \in Z$, then

$$
\|c\|_{l_{p}}+\|d\|_{l_{p, q}^{s}} \preceq\|f\|_{B_{p, q}^{s}(R)}
$$

for $f \in B_{p, q}^{s}(R), 0<s<m$.

\section{Proof of Main Theorem}

This section is devoted to proving the Main Theorem. We begin with three lemmas for proving upper and lower bounds of the characterization.

Lemma 2.1 Let $p \in\left(\frac{2}{3}, 2\right], q \in(0, \infty], s>0$ be arbitrary, then $\left\|\phi_{0}\right\|_{B_{p, q}^{s}} \leq C$ and $\left\|\psi_{j}\right\|_{B_{p, q}^{s}} \leq$ $C$ with a uniform constant $C>0$ for $j \in N_{0}$.

Proof First, we will show $\left\|\phi_{j}\right\|_{p} \leq C$ with a uniform constant $C$ for all $j$. By Lemma 2.1, Theorem 2.8 in [2], and Theorem 2.1 in [11], $\phi_{j}$ are all compactly supported and $\operatorname{Supp} \phi_{j} \subset$ $[-L, L]$ for a uniform constant $L>0$. Therefore, Supp $\psi_{j} \subset[-L, L]$ for all $j$ because of $\widehat{\psi}_{j-1}(\xi):=\widehat{b}_{j}^{1}\left(\frac{\xi}{2}\right) \widehat{\phi}_{j}\left(\frac{\xi}{2}\right)=e^{-i \xi} \widehat{\widehat{a}}_{j}(\xi+\pi) \widehat{\phi}_{j}\left(\frac{\xi}{2}\right)$. Note that $\left\|\phi_{j}\right\|_{L_{2}} \leq 1$ (Lemma 2.2 in [2]). This with

$$
\begin{aligned}
& {\left[\int\left|\phi_{j}(x)\right|^{p} d x\right]^{\frac{1}{p}}} \\
& \quad \leq\left\{\left[\int\left(\frac{1}{\left(1+x^{2}\right)^{p}}\right)^{\frac{2}{2-p}} d x\right]\left[\int\left(\left(1+x^{2}\right)^{p}\left|\phi_{j}(x)\right|^{p}\right)^{\frac{2}{p}} d x\right]^{\frac{p}{2}}\right\}^{\frac{1}{p}} \\
& \quad \leq C_{p, 2}\left\|\phi_{j}\right\|_{L_{2}}
\end{aligned}
$$

for $\frac{2}{3}<p \leq 2$ leads to $\left\|\phi_{j}\right\|_{L_{p}} \leq C$ for all $j \in N_{0}$. $\left\|\psi_{j}\right\|_{L_{p}} \leq C$ holds similarly.

Second, let $[s]+1=M$, by $(2.1)$,

$$
\begin{aligned}
& \left\|\phi_{0}\right\|_{B_{p, q}^{s}} \\
& \quad=\left\|\phi_{0}\right\|_{p}+\left\|\left(2^{l s} \omega_{p}^{M}\left(\phi_{0}, 2^{-l}\right)\right)_{l \in Z}\right\|_{l_{q}} \\
& \quad \preceq\left\|\phi_{0}\right\|_{p}+\left\|2^{l(s-M)}\right\|_{l_{q}}\left\|\phi_{0}^{(M)}\right\|_{p} \\
& \quad \preceq\left\|\phi_{0}\right\|_{p}+\left\|\phi_{0}^{(M)}\right\|_{2} \leq\left\|\phi_{0}\right\|_{H_{2}^{M}} .
\end{aligned}
$$

Note that

$$
\left\|\phi_{0}\right\|_{H_{2}^{M}}^{2} \preceq \sum_{k}\left|\left\langle\phi_{0}, \phi_{0 ; 0, k}\right\rangle\right|^{2}+\sum_{j=0}^{\infty} \sum_{k} 2^{2 j s}\left|\left\langle\phi_{0}, \psi_{j ; j, k}\right\rangle\right|^{2}=1
$$


because of Corollary 3.3 in [1] and the orthonormality property of $\left.\left\{\phi_{0 ; 0, k}\right\}_{k},\left\{\psi_{j ; j, k}\right\rangle\right\}_{j, k}$. This with (2.1) leads to

$$
\left\|\phi_{0}\right\|_{B_{p, q}^{s}} \leq C
$$

as well as $\left\|\psi_{j}\right\|_{B_{p, q}^{s}} \leq C$ for $j \in N_{0}$ with a uniform constant $C>0$. Thus, the result holds.

The second lemma comes from [5], Lemma 3.4 and the third one comes from [6], (3.2.26).

Lemma 2.2 Suppose $\varphi \in B_{p, \infty}^{\sigma}\left(R^{n}\right)$ is compactly supported with $0<p, q \leq \infty$ and $0<s<\sigma$. Then

$$
\left\|\sum_{k \in Z^{n}} c_{k} \varphi_{j, k}(\cdot)\right\|_{B_{p, q}^{s}\left(R^{n}\right)} \preceq 2^{\left(\sigma+\frac{n}{2}-\frac{n}{p}\right) j}\|c\|_{\ell_{p}}, \quad\left\|\sum_{j=j_{0}}^{\infty} \sum_{k \in Z^{n}} d_{j, k} \varphi_{j, k}(\cdot)\right\|_{B_{p, q}^{s}\left(R^{n}\right)} \preceq\|d\|_{\ell_{p, q}^{s}} .
$$

Note that the constants are uniform because of $\|\varphi\|_{B_{p, \infty}^{\sigma}\left(R^{n}\right)} \leq C$.

Lemma 2.3 If $f \in L_{p}(R), 1 \leq p \leq \infty$, define $\sigma$ as a closed interval of $R$ and let $P_{m-1}$ be the set of m-order polynomials, then

$$
\inf _{P \in \Pi_{m-1}}\|f-P\|_{L_{p}\left(\sigma_{j, k}\right)} \preceq \omega_{p}^{m}\left(f, 2^{-j}, \sigma_{j, k}\right)
$$

where $\sigma_{j, k}:=2^{-j}(\sigma+k)$ and $\left.\left.\omega_{p}^{m}\left(f, 2^{-j}, \sigma_{j, k}\right):=\sup _{|h| \leq 2^{-j}}\left\|\Delta_{h}^{M} f(\cdot)\right\|_{L_{p}\left(\left(\sigma_{j, k}\right)\right.}\right)_{h, M}\right)$ with $\left(\sigma_{j, k}\right)_{h, M}:=$ $\left\{x \in \sigma_{j, k}, x+l h \in \sigma, l=1,2, \ldots, M\right\}$.

Now, we are in the position to show the Main Theorem.

By Lemma 2.1, $\left\|\phi_{0}\right\|_{B_{p, q}^{s}} \leq C$ and $\left\|\psi_{j}\right\|_{B_{p, q}^{s}} \leq C$. This, with Lemma 2.2, shows that

$$
\begin{aligned}
& \left\|\sum_{k} c_{0, k} \phi_{0 ; 0, k}+\sum_{j=0}^{\infty} \sum_{k \in Z} d_{j, k} \psi_{j ; j, k}\right\|_{B_{p, q}^{s}(R)} \\
& \quad \leq\left\|\sum_{k} c_{0, k} \phi_{0 ; 0, k}\right\|_{B_{p, q}^{s}(R)}+\left\|\sum_{j=0}^{\infty} \sum_{k \in Z} d_{j, k} \psi_{j ; j, k}\right\|_{B_{p, q}^{s}(R)} \\
& \quad \preceq\|c\|_{\ell_{p}}+\|d\|_{\ell_{p, q}^{s}} .
\end{aligned}
$$

To prove the lower bound, one finds that $\left|\left\langle f, \phi_{0 ; 0, k}\right\rangle\right| \leq\|f\|_{L_{p}\left(\sigma_{0, k}\right)}$,

$$
\sum_{k}\left|\left\langle f, \phi_{0 ; 0, k}\right\rangle\right|^{p} \preceq \sum_{k} \int_{\sigma_{0, k}}|f(x)|^{p} d x \preceq\|f\|_{p}^{p},
$$

where $\sigma_{0, k}:=\operatorname{Supp} \phi_{0 ; 0, k}$. Let $\sigma_{j, k}:=\operatorname{Supp} \psi_{j ; j, k}$. This with $m:=\inf _{j} m_{j+1}$ shows that

$$
\begin{aligned}
& \left|\left\langle f, \psi_{j ; j, k}\right\rangle\right|^{p} \\
& \quad=\inf _{P \in P_{m-1}}\left|\left\langle f-P, \psi_{j ; j, k}\right\rangle\right|^{p}
\end{aligned}
$$




$$
\begin{aligned}
& \preceq \inf _{P \in P_{m-1}}\|f-P\|_{L_{p}\left(\sigma_{j, k}\right)}^{p} 2^{\frac{j p}{2}-\frac{j p}{p^{\prime}}} \\
& \leq 2^{\frac{j p}{2}-\frac{j p}{p^{\prime}}} \omega_{p}^{m}\left(f, 2^{-j}, \sigma_{j, k}\right),
\end{aligned}
$$

where the equality comes from the $m_{j+1}$ vanishing moments of $\psi_{j}$ by Theorem 1.1 and the second inequality holds due to Lemma 2.3. Then $\left\|\left(d_{j, k}\right)_{k}\right\|_{l_{p}} \preceq 2^{\frac{j}{2}-\frac{j}{p^{\prime}}} \omega_{p}^{m}\left(f, 2^{-j}\right)$ by the same proof as of (3.3) in [8]. Therefore,

$$
\|d\|_{l_{p, q}^{s}} \preceq\left\|\left(2^{s+\frac{j}{2}-\frac{j}{p^{\prime}}} 2^{\frac{j}{2}-\frac{j}{p^{\prime}}} \omega_{p}^{m}\left(f, 2^{-j}\right)\right)_{j \in Z}\right\|_{l_{q}} \leq\|f\|_{B_{p, q}^{s}(R)} .
$$

Remark 2.1 In conclusion, we have a characterization of Besov spaces by

$$
\|f\|_{B_{p, q}^{s}} \sim\|c\|_{l_{p}}+\|d\|_{l_{p, q}^{s}}
$$

with $c_{0, k}:=\left\langle f, \phi_{0 ; 0, k}\right\rangle, d_{j, k}:=\left\langle f, \psi_{j ; j, k}\right\rangle, 1 \leq p \leq 2,0<q \leq \infty, 0<s<m$, and $f \in B_{p, q}^{s}(R)$.

Remark 2.2 Some questions are left to be considered. Note that we assume $1 \leq p \leq 2$ in our Main Theorem. Then a natural question is to study the case for $p>2$. Another one is to discuss whether or not the wavelet frames of (i) in Theorem 1.1 can characterize Besov spaces. The last question is to relax the restriction $s \in(0, m)$.

\section{Competing interests}

The author declares that he has no competing interests.

\section{Acknowledgements}

The author would like to thank the referees for their helpful comments. This work is supported by the National Natural Science Foundation of China, Tian Yuan Foundation (Grant No. 11226106) and the National Natural Science Foundation of China (Grant No. 11201094)

Received: 25 December 2013 Accepted: 16 April 2014 Published: 02 May 2014

\section{References}

1. Han, B, Shen, Z: Characterization of Sobolev spaces of arbitrary smoothness using nonstationary tight wavelet frames. Isr. J. Math. 172, 371-398 (2009)

2. Han, B, Shen, Z: Compactly supported symmetric $C^{\infty}$ wavelets with spectral approximation order. SIAM J. Math. Anal. 40, 905-938 (2009)

3. Hans, T: Theory of Function Spaces II. Birkhäuser, Basel (1992)

4. Härdle, W, Kerkyacharian, G, Picard, D, Tsybakov, A: Wavelets, Approximation and Statistal Applications. Springer, Berlin (1998)

5. Bittner, K, Urban, K: On interpolatory divergence-free wavelets. Math. Comput. 76(258), 903-929 (2007)

6. Cohen, A: Wavelet methods in numerical analysis. In: Handbook of Numerical Analysis. Handb. Numer. Anal., vol. VII, pp. 417-711. North-Holland, Amsterdam (2000)

7. Liu, Y, Zhao, J: Convergence of Hermite interpolatory operators. Sci. China Math. 53(8), 2115-2126 (2010)

8. Zhao, J: Interpolatory Hermite splines on rectangular domains. Appl. Math. Comput. 216, 2799-2813 (2010). doi:10.1016/j.amc.2010.03.130

9. Liu, Y, Zhao, J: An extension of Bittner and Urban's theorem. Math. Comput. 82(281), 401-411 (2013)

10. Jia, RQ, Wang, JZ, Zhou, DX: Compactly supported wavelets bases for Sobolev spaces. Appl. Comput. Harmon. Anal. $15,224-241(2003)$

11. Cohen, A, Dyn, N: Nonstationary subdivision schemes and multiresolution analysis. SIAM J. Math. Anal. 27, 1745-1769 (1996) 